\title{
Ventilação mandatória intermitente sincronizada versus ventilação com suporte pressórico e volume garantido em coelhos induzidos à hemorragia aguda
}

[Synchronized intermittent mandatory ventilation versus volume assured pressure support ventilation in rabbits induced to acute hemorrhage]

P.A.C.S. Batista ${ }^{1}$, N. Nunes ${ }^{2}$, A.A. Camacho ${ }^{2}$, P.A. Borges ${ }^{1}$, J.V. Moro ${ }^{1}$, P.C.F. Lopes $^{3}$, C.P. Burger ${ }^{1}$, F. N. Gava

${ }^{1}$ Aluna de pós-graduação - Faculdade de Ciências Agrárias e Veterinárias - FCAV-UNESP - Jaboticabal, SP ${ }^{2}$ Faculdade de Ciências Agrárias e Veterinárias - FCAV-UNESP - Jaboticabal, SP

${ }^{3}$ Pós-doutoranda - Faculdade de Ciências Agrárias e Veterinárias - FCAV-UNESP - Jaboticabal, SP

\section{RESUMO}

Avaliaram-se os efeitos da ventilação mandatória intermitente sincronizada (SIMV) e da ventilação com pressão de suporte e volume garantido (VAPSV) sobre os parâmetros cardiorrespiratórios em coelhos anestesiados com propofol e submetidos à hipovolemia aguda. Vinte animais da raça Nova Zelândia foram distribuídos aleatoriamente em dois grupos: GM sob SIMV e GV sob VAPSV. Na medicação pré-anestésica, foram administradas quetamina $(15 \mathrm{mg} / \mathrm{kg})$ e xilazina $(1 \mathrm{mg} / \mathrm{kg})$ intramuscular. O propofol foi administrado na indução $(8 \mathrm{mg} / \mathrm{kg})$ e na manutenção anestésica $(0,5 \mathrm{mg} / \mathrm{kg} / \mathrm{min})$. Em seguida, em cada grupo foi iniciada a modalidade ventilatória. Após 30 minutos da indução, os coelhos foram submetidos à hipovolemia, retirandose $12 \mathrm{~mL} / \mathrm{kg}$ de sangue arterial. A primeira mensuração das características (M0) foi efetuada 40 minutos após a indução anestésica, seguida de mensurações em intervalos de 10 minutos depois da hipovolemia (M1 a M6). A partir de M3, o débito cardíaco foi maior em GM. Em ambos os grupos, as pressões arteriais e pressão venosa central diminuíram a partir de M1, enquanto a pressão arterial de oxigênio no sangue arterial aumentou a partir de M4. O esforço respiratório foi maior no GV em todos os momentos estudados. Conclui-se que a SIMV e a VAPSV foram seguras quanto à oxigenação arterial, garantindo uma adequada troca gasosa. Contudo, a SIMV mostrou-se a mais indicada em coelhos hipovolêmicos por manter melhor a estabilidade hemodinâmica, com a vantagem de proporcionar menor trabalho respiratório.

Palavras-chave: coelhos, anestesia intravenosa, hipovolemia, ventilação mecânica

\begin{abstract}
The effects of synchronized intermittent mandatory ventilation (SIMV) versus volume assured pressure support ventilation (VAPSV) on cardiorespiratory parameters in propofol-anesthetized rabbits induced to acute hypovolemia were evaluated. Twenty New Zealand white rabbits were randomly allotted to: GM under SIMV and GV under VAPSV. In premedication, ketamine $(15 \mathrm{mg} / \mathrm{kg})$ and xylazine $(1 \mathrm{mg} / \mathrm{kg})$ were administered intramuscularly. Propofol was used to induce $(8 \mathrm{mg} / \mathrm{kg})$ and to maintain anesthesia $(0.5 \mathrm{mg} / \mathrm{kg} / \mathrm{min})$. Following, according to each group, the ventilation mode was started. After thirty minutes of anesthesia induction, rabbits were induced to hypovolemia by removing $12 \mathrm{ml} / \mathrm{kg}$ of arterial blood. The initial measurement of parameters (MO) was recorded thirty minutes after anesthesia induction. Additional recordings were performed at 10minute intervals after hypovolemia induction (M1 to M6). Cardiac output (CO) was bigger in GM. In both groups, arterial pressures and central venous pressure $(C V P)$ decreased from $M 1$, while arterial partial pressure of oxygen $\left(\mathrm{PaO}_{2}\right)$ increased from $M 4$. The respiratory effort was greater in $G V$ at all times studied. In conclusion, VAPSV and SIMV were safe for arterial oxygenation and provided adequate gas exchange. However, the SIMV is more appropriate for hypovolemic rabbits, because it maintains hemodynamic stability and promotes lower respiratory work.
\end{abstract}

Keywords: rabbits. hypovolemia, intravenous anesthesia, mechanical ventilation

Recebido em 9 de maio de 2011

Aceito em 3 de setembro de 2012

E-mail: priacs@hotmail.com 


\section{INTRODUÇÃO}

$\mathrm{Na}$ rotina da clínica veterinária de pequenos animais, é alta a incidência de casos de traumas com hemorragias, ocasionando quadro de hipovolemia, que é definida como uma anormalidade no sistema circulatório que resulta em perfusão e oxigenação tecidual inadequada (Pepe et al., 2004). Assim, nesses pacientes hipovolêmicos, muitas vezes é necessário instituir um suporte respiratório por meio de ventilação mecânica (VM). Isso porque ocorre aumento do trabalho respiratório (Wobvt), o que em casos extremos pode consumir até $25 \%$ da oferta de oxigênio aos tecidos periféricos, resultando em hipoxemia (Fink, 1996).

Todavia, durante a ventilação mecânica, ocorre a diminuição do retorno venoso e do débito cardíaco (DC), que podem acentuar a hipotensão desses pacientes (Barbas et al., 1998). Dessa maneira, com a indicação de corrigir a hipoxemia, sem causar alteração hemodinâmica concomitante, recomenda-se a utilização de técnicas de VM associadas com respiração espontânea (Romaldini, 2006).

A ventilação mandatória intermitente sincronizada (SIMV) caracteriza-se por intercalar ciclos mandatórios com períodos variáveis de respiração espontânea (Manczur e Greenough, 2000). Esse suporte ventilatório permite reduzir a pressão média das vias aéreas (MAP) quando comparada a outras técnicas, resultando em um melhor retorno venoso e desempenho cardíaco (Neumann et al., 2005).

A ventilação com pressão de suporte e volume assegurado (VAPSV) combina a pressão de suporte e a ventilação ciclada a volume em um mesmo ciclo respiratório (Emmerich et al., 2001). Essa técnica é considerada um modo de duplo-controle, pois alterna o mecanismo de ciclagem entre pressão e volume, caso o volume corrente mínimo programado não seja atingido pela pressão inspiratória máxima pré-fixada, por meio de um sistema duplo de fluxo (Emmerich, 2008).

Quando se opta pela ventilação mecânica, devem-se buscar fármacos que proporcionem estabilidade cardiovascular. Nessas situações, o propofol merece destaque, pois, durante sua infusão contínua, há estabilidade dos parâmetros hemodinâmicos (Lopes et al., 2009). Adicionalmente, esse agente intravenoso proporciona rápida indução e recuperação (Nolan, 2006), além de ser rapidamente metabolizado (Deneuche e Desbois, 1999). Por ser isento de efeito cumulativo, pode ser usado na forma de bolus, para indução e na manutenção anestésica em infusão contínua (Aguiar et al., 2001).

Assim, este estudo tem por objetivo avaliar os efeitos cardiovasculares e hemogasométricos da ventilação mandatória intermitente sincronizada e da ventilação com pressão de suporte e volume garantido em coelhos anestesiados com infusão contínua de propofol e submetidos à hipovolemia aguda.

\section{MATERIAL E MÉTODOS}

Este trabalho foi aprovado pelo Comitê de Ética e Bem-Estar Animal, processo ${ }^{\circ} 025474$. Foram utilizados 20 coelhos da raça Nova Zelândia, adultos, machos, pesando $4,2 \pm 0,1 \mathrm{~kg}$, hígidos, provenientes de produtor especializado na criação da espécie. Os animais foram distribuídos, aleatoriamente, em dois grupos experimentais, constituídos de dez coelhos cada, denominados: grupo ventilação com pressão de suporte de volume garantido (GV) e grupo ventilação mandatória intermitente sincronizada (GM).

Os coelhos não foram submetidos a jejum alimentar e hídrico, visto que tal procedimento não é necessário nessa espécie (Flecknell, 2007). Os animais receberam como medicação pré-anestésica xilazina, $1 \mathrm{mg} / \mathrm{kg}$, e quetamina, $15 \mathrm{mg} / \mathrm{kg}$, pela via intramuscular. Transcorridos 20 minutos, induziu-se a anestesia geral pela administração intravenosa de propofol, $8 \mathrm{mg} / \mathrm{kg}$. Em seguida, iniciou-se a infusão contínua do mesmo fármaco, por meio de bomba de infusão, na dose de $0,5 \mathrm{mg} / \mathrm{kg} / \mathrm{min}$.

Para a intubação orotraqueal, os animais foram posicionados em decúbito lateral direito, com o pescoço em hiperextensão, e a sonda traqueal de diâmetro $3 \mathrm{~cm}$ foi introduzida na cavidade oral, sendo utilizada a técnica "às cegas" segundo descrita por Alexander e Clark (1980). Logo após, os coelhos foram posicionados em decúbito lateral direito, sobre colchão térmico ativo, no qual permaneceram por todo o período 
experimental, mantendo-se a temperatura corpórea em $38,6 \pm 5^{\circ} \mathrm{C}$. Após a estabilização da anestesia, procedeu-se à modalidade ventilatória de acordo com cada grupo. Para os coelhos do GM foi ajustada a frequência respiratória em 12 movimentos/min, fluxo inspiratório 1,2L e pressão $12 \mathrm{mmHg}$, e do $\mathrm{GV}$, frequência respiratória em 20 movimentos/minuto, pressão de suporte $10 \mathrm{cmH}_{2} \mathrm{O}$, fluxo inspiratório $20 \mathrm{~L} / \mathrm{min}$ e volume $0,5 \mathrm{~L}$. Para ambos os grupos, a fração inspirada de $\mathrm{O}_{2}$ foi de $60 \%$, a relação inspiração expiração 1:2 e a sensibilidade do ventilador foi ajustada em $-0,5 \mathrm{cmH}_{2} \mathrm{O}$.

A frequência respiratória (FR), pressão média das vias aéreas (MAP) e trabalho respiratório (Wobvt) foram monitorados continuamente pelo monitor de mecânica respiratória (Monitor Dixtal DX 8.100 Intermed - Manaus, AM, Brasil Processo FAPESP 03/11.125/7), cujo sensor foi conectado à sonda orotraqueal.

Em seguida, foi realizada incisão na região do trígono femoral direito para exposição da artéria femoral, na qual foi introduzido um cateter de calibre $22 \mathrm{G}$, para mensuração da pressão arterial (Dixtal - Mod. Dx2010, Módulo de PA invasiva, Manaus - AM, Brasil - Processo FAPESP 02/04625-0) e para posterior coleta de sangue para hemogasometria (Hemogasometro Omni C. Roche Diagnostics GmbH. Mannheim, Alemanha - Processo FAPESP 02/14054-0) com a finalidade de se obter a saturação de oxihemoglobina $\left(\mathrm{SaO}_{2}\right)$, déficit de base (DB), bicarbonato $\left(\mathrm{HCO}_{3}{ }^{-}\right), \mathrm{pH}$, pressão parcial de oxigênio no sangue arterial $\left(\mathrm{PaO}_{2}\right)$ e de dióxido de carbono $\left(\mathrm{PaCO}_{2}\right)$. Após 30 minutos da indução anestésica, os animais foram submetidos à hipovolemia aguda, retirando-se sangue arterial (12mL kg-1) num período de cinco minutos. Concomitantemente, foi realizada incisão na pele, na região cervical, sobre a veia jugular esquerda, com sua posterior exposição, para introdução de um cateter de polietileno (Sonda uretral de PVC n ${ }^{\circ}$ 04, Embramed Ind. Com. Ltda, São Paulo, SP, Brasil), com o intuito de mensurar a pressão venosa central (PVC).

A frequência cardíaca (FC) foi obtida por eletrocardiógrafo computadorizado (TEB Mod. ECG PC - software versão 1.10, São Paulo - SP, Brasil - Processo FAPESP 96/1151-5), ajustado para leitura na derivação DII. Por meio da ecodopplercardiografia transtorácica, registraram-se os valores de débito cardíaco (DC). Para tanto, utilizou-se o eletrocardiógrafo (TEB - Mod. ECG PC software versão 1.10, São Paulo - SP,Brasil Processo FAPESP 96/1151-5) com transdutor bifrequencial de $5,0-7,5 \mathrm{MHz}$, que foi posicionado na janela paraesternal esquerda, entre o quarto e o quinto espaço intercostal, para colheita da imagem espectral bidimensional do fluxo sanguíneo pulmonar, utilizando o recurso Doppler pulsado.

As primeiras mensurações foram realizadas quarenta minutos após a indução anestésica (M0) e, em seguida, induziu-se a hipovolemia como descrito anteriormente. Decorridos dez minutos após a indução da hemorragia, realizou-se nova mensuração (M1), seguindo-se mensurações realizadas em intervalos de dez minutos (M2, M3, M4, M5 e M6) durante 60 minutos.

Os dados obtidos foram analisados pelo método dos quadrados mínimos, utilizando o procedimento GLM do programa computacional SAS. Nos resultados em que foram verificadas diferenças significativas entre os tratamentos $(\mathrm{P}<0,05)$, as médias foram comparadas pelo teste Tukey - Kramer, a 5\% de probabilidade.

Os coelhos foram submetidos à eutanásia no término do experimento, após a administração intravenosa de propofol na dose de $16 \mathrm{mg} / \mathrm{kg}$, seguido de $40 \mathrm{~mL}$ de solução de cloreto de potássio a $19,1 \%$, pela via intravenosa, depois de se constatar que os animais estavam no estágio quatro de Guedel, indicando o aprofundamento do plano anestésico.

\section{RESULTADOS E DISCUSSÃO}

A FC (Tab.1) permaneceu estável e próxima aos limites considerados fisiológicos para a espécie, 198 - $300 \mathrm{bat} / \mathrm{min}$, em ambos os grupos, e não foi observada diferença significativa entre eles (Suckow e Douglas, 1996). A estabilidade registrada para a característica permite sugerir a ausência da resposta compensatória à diminuição da volemia, que é caracterizada pelo aumento da FC após perdas excessivas de sangue (Haskins, 2005). Resultado semelhante foi observado por Moro (2009), que não verificou aumento da FC após a indução da hipovolemia em coelhos anestesiados com isofluorano e tramadol e mantidos em ventilação espontânea. $\mathrm{O}$ motivo do 
fracasso dos mecanismos compensatórios em resposta à hipotensão induzida neste estudo pode ser devido à vasodilatação e ao inotropismo negativo (Baurmet et al., 2005), que são induzidos pela maioria dos anestésicos, inclusive pelo propofol (Reves, 2000).

Neste estudo, antes da indução da hipovolemia, a pressão arterial média estava abaixo do valor fisiológico para a espécie (entre 85 e 110 $\mathrm{mmHg}$ ), segundo Suckow e Douglas (1996). Contudo, esses valores são descritos para animais despertos e, em M0, os coelhos encontravam-se anestesiados e sob VM. Assim, tal evento pode ser atribuído ao propofol, o qual possui efeito vasodilatador em coelhos com consequente redução da PA (Royse et al., 2008). Esse resultado corrobora Borges et al. (2011), que, em estudo com coelhos hipovolêmicos, anestesiados com propofol e submetidos a ventilação mecânica controlada à pressão, observaram médias de PAM menores que 85 $\mathrm{mmHg}$. No entanto, considerando os valores de $\mathrm{PaO}_{2}, \mathrm{PaCO}_{2}$ e $\mathrm{SaO}_{2}$ obtidos neste estudo, aventa-se afirmar que este achado não teve relevância clínica, pois não houve comprometimento da troca gasosa nos alvéolos, já que as médias obtidas para esses parâmetros situaram-se dentro da faixa fisiológica para a espécie.

Por outro lado, os valores de PAS, PAD, PAM e PVC em M1 diminuíram (Tab.1) em razão de alteração do volume sanguíneo e consequente diminuição do retorno venoso, em ambos os grupos. Esses resultados são condizentes com os de Hirano et al. (2003), que observaram diminuição da PAM após o choque experimental, em ratos anestesiados com quetamina e xilazina. Todavia, em GV e GM, nos demais momentos, as pressões arteriais e PVC permaneceram estáveis, indicando que a utilização de diferentes modalidades ventilatórias não alterou esses parâmetros. Apesar de ser descrito que a diminuição do retorno venoso da pressão arterial e consequente PVC são as alterações hemodinâmicas mais importantes durante a ventilação controlada (Romaldini, 2006), neste estudo não foram constatados tais achados, pois todas as mensurações foram realizadas quando a ventilação mecânica já estava instituída.
O DC diminuiu a partir de M3 em ambos os grupos (Tab. 1), devido à indução da hipovolemia, pois o choque hipovolêmico é caracterizado por ocasionar redução do DC, levando a baixa perfusão tecidual (Pepe et al., 2004). Adicionalmente, sabe-se que o fluxo sanguíneo do pulmão é igual ao débito cardíaco, assim, sob as condições de pressão alveolar de oxigênio $\left(\mathrm{PAO}_{2}\right)$ e $\mathrm{DC}$ de repouso, a transferência de oxigênio do alvéolo para o capilar pulmonar é limitada pela perfusão (Levitzky, 2004). Dessa forma, justifica-se a importância clínica das alterações do DC para o sistema respiratório. Todavia, neste estudo, verificaram-se maiores médias de DC nos animais que foram submetidos à SIMV, as quais podem ser justificadas pela característica dessa modalidade em melhorar a função cardíaca, por meio da diminuição da pressão intrapleural, ocasionando assim aumento do retorno venoso e manutenção do DC (Matsumoto e Almeida, 2005). Diante disso, pode-se afirmar que a SIMV é uma técnica de suporte ventilatório que não ocasiona alteração hemodinâmica significativa, permitindo maior eficiência nas trocas gasosas nos alvéolos

A SIMV e a VAPSV são modalidades ventilatórias que permitem ao paciente realizar respirações espontâneas nos intervalos dos ciclos programados (Martins et al., 2005). A FR estabelecida no GM foi de $15 \mathrm{mov} / \mathrm{min}$, enquanto que no grupo $\mathrm{GV}$ foi ajustada a FR em 20 mov/min. No entanto, pode-se notar que, em todos os momentos, esse parâmetro permaneceu acima dos valores fixados em ambos os grupos (Tab. 1), indicando que os coelhos respiraram espontaneamente entre os intervalos das ventilações controladas.

Para a FR não houve diferença significativa entre os grupos nem entre os momentos (Tab.1), permanecendo as médias próximas aos valores considerados fisiológicos para a espécie, 30 a 60mov/min (Suckow e Douglas, 1996), indicando que ambas as modalidades ventilatórias foram capazes de garantir a ventilação dos animais durante $\mathrm{o}$ modelo experimental estudado. 
Ventilação mandatória intermitente...

Tabela 1. Valores médios e desvios padrão $(\mathrm{x} \pm \mathrm{s})$ de frequência cardíaca $(\mathrm{FC})$, pressões arteriais sistólica (PAS), diastólica (PAD) e média (PAM), pressão venosa central (PVC), débito cardíaco (DC), frequência respiratória (FR), pressão média das vias aéreas (MAP) e trabalho respiratório (Wobvt) obtidos em coelhos submetidos à hipovolemia aguda, anestesiados com propofol $(0,5 \mathrm{mg} / \mathrm{kg} / \mathrm{min})$ e ventilados com SIMV (GM) ou VAPSV (GV).

\begin{tabular}{|c|c|c|c|c|c|c|c|c|}
\hline \multirow[b]{2}{*}{ Parâmetro } & \multirow[b]{2}{*}{ Grupo } & \multicolumn{7}{|c|}{ Momentos } \\
\hline & & M0 & M1 & M2 & M3 & M4 & M5 & M6 \\
\hline $\mathrm{FC}$ & GM & $182 \pm 11$ & $179 \pm 16$ & $178 \pm 16$ & $176 \pm 17$ & $178 \pm 26$ & $181 \pm 24$ & $183 \pm 17$ \\
\hline (bat./min.) & GV & $195 \pm 23$ & $194 \pm 22$ & $190 \pm 13$ & $186 \pm 23$ & $185 \pm 17$ & $186 \pm 24$ & $185 \pm 20$ \\
\hline PAS & GM & $71 \pm 19 a$ & $37 \pm 20 b$ & $40 \pm 24 b$ & $42 \pm 27 b$ & $43 \pm 26 b$ & $42 \pm 27 b$ & $40 \pm 24 b$ \\
\hline$(\mathrm{mmHg})$ & GV & $70 \pm 32 a$ & $34 \pm 20 b$ & $35 \pm 21 b$ & $37 \pm 13 b$ & $38 \pm 22 b$ & $38 \pm 21 b$ & $38 \pm 21 b$ \\
\hline PAD & GM & $49 \pm 30 a$ & $24 \pm 12 b$ & $31 \pm 27 b$ & $29 \pm 18 b$ & $32 \pm 27 b$ & $32 \pm 9 b$ & $30 \pm 25 b$ \\
\hline$(\mathrm{mmHg})$ & GV & $57 \pm 21 a$ & $30 \pm 23 b$ & $30 \pm 21 b$ & $33 \pm 23 b$ & $32 \pm 22 b$ & $33 \pm 22 b$ & $33 \pm 20 b$ \\
\hline PVC & GM & $0 \pm 2 \mathrm{a}$ & $-2 \pm 4 b$ & $-1 \pm 3 b c$ & $-1 \pm 2 b c$ & $-1 \pm 2 b c$ & $-1 \pm 2 b c$ & $-2 \pm 2 b c$ \\
\hline (mmHg) & GV & $1 \pm 2 \mathrm{a}$ & $-1 \pm 2 b$ & $-2 \pm 3 b c$ & $-2 \pm 3 b c$ & $-2 \pm 2 b c$ & $-2 \pm 2 b c$ & $-2 \pm 3 b c$ \\
\hline DC & GM & $0,32 \pm 0,03$ & $0,32 \pm 0,03$ & $0,31 \pm 0,01$ & $0,30 \pm 0,02 \mathrm{~A}$ & $0,30 \pm 0,02 \mathrm{~A}$ & $0,30 \pm 0,02 \mathrm{~A}$ & $0,29 \pm 0,03 \mathrm{~A}$ \\
\hline (L/min) & GV & $0,36 \pm 0,03 \mathrm{a}$ & $0,34 \pm 0,03 a$ & $0,31 \pm 0,03 b$ & $0,25 \pm 0,02 \mathrm{Bbc}$ & $0,21 \pm 0,02 \mathrm{Bc}$ & $0,20 \pm 0,02 \mathrm{Bc}$ & $0,19 \pm 0,01 \mathrm{Bc}$ \\
\hline$(\mathrm{cmH} 2 \mathrm{O})$ & GV & $4 \pm 0,5$ & $4 \pm 0,9$ & $4 \pm 0,8$ & $4 \pm 0,6$ & $4 \pm 0,8$ & $4 \pm 0,5$ & $4 \pm 0,5$ \\
\hline Wobvt & GM & $0,7 \pm 0,1 \mathrm{~A}$ & $0,7 \pm 0,2 \mathrm{~A}$ & $0,7 \pm 0,2 \mathrm{~A}$ & $0,7 \pm 0,1 \mathrm{~A}$ & $0,7 \pm 0,2 \mathrm{~A}$ & $0,7 \pm 0,2 \mathrm{~A}$ & $0,7 \pm 0,1 \mathrm{~A}$ \\
\hline$(\mathrm{J} / \mathrm{L})$ & GV & $2 \pm 0,1 \mathrm{~B}$ & $2 \pm 0,3 \mathrm{~B}$ & $2 \pm 0,1 \mathrm{~B}$ & $2 \pm 0,3 \mathrm{~B}$ & $2 \pm 0,3 \mathrm{~B}$ & $2 \pm 0,1 \mathrm{~B}$ & $2 \pm 0,2 \mathrm{~B}$ \\
\hline
\end{tabular}

Médias seguidas por letras maiúsculas diferentes, nas colunas, diferem entre si com $\mathrm{P}<0,05$.

Médias seguidas por letras minúsculas diferentes, nas linhas, diferem entre si com $\mathrm{P}<0,05$.

A MAP apresenta correlação direta com a ventilação alveolar, oxigenação arterial e desempenho hemodinâmico (David, 2001). Neste estudo não houve diferenças significativas da MAP quando comparada às modalidades ventilatórias.

A SIMV nesta pesquisa mostrou-se capaz de reduzir o Wobvt em relação ao modo VAPSV. Essa diferença pode ser explicada através do conceito das modalidades. A VAPSV combina a pressão de suporte e a ventilação ciclada a volume num mesmo ciclo respiratório (Bonassa e Amato, 1995), enquanto a SIMV caracteriza-se por intercalar ciclos mandatórios com períodos variáveis de respiração espontânea (Martins et al., 2005). Dessa forma, atribui-se essa diferença à presença dos ciclos espontâneos na SIMV. Neste estudo, as duas técnicas ventilatórias garantiram uma troca gasosa adequada. Assim, deve-se buscar uma melhor interação entre o paciente e o ventilador, pois a assincronia gerada pelo Wobvt em excesso pode acarretar um gasto desnecessário de energia. Isso porque tal evento proporciona maior consumo de oxigênio, ocasionando um comprometimento hemodinâmico ainda mais acentuado aos pacientes hipovolêmicos (Ferreira et al., 2005).
Diante desses comentários, pode-se atribuir uma vantagem à utilização da SIMV em condições de hipovolemia.

A $\mathrm{PaO}_{2}$ exprime a eficácia das trocas de oxigênio entre os alvéolos e os capilares pulmonares, e depende diretamente da $\mathrm{FiO}_{2}$, modalidade ventilatória empregada e da relação ventilaçãoperfusão pulmonar (Cortopassi et al., 2002). Para coelhos hígidos, respirando ar ambiente, a $\mathrm{PaO}_{2}$ prevista é de aproximadamente $90 \mathrm{mmHg}$ (Barzago et al., 1992). Dessa forma, as médias registradas neste estudo, de 117 a $183 \mathrm{mmHg}$ (Tab. 2), permaneceram acima da faixa fisiológica para a espécie, pois a $\mathrm{FiO}_{2}$ empregada foi de 0,6. Assim sendo, esses resultados confirmam os de Figueiredo et al. (2003), que estudaram a hipotensão induzida em coelhos anestesiados com halotano e mantidos em ventilação espontânea e registraram médias de $\mathrm{PaO}_{2}$ entre 110 e $178 \mathrm{mmHg}$. Portanto, pode-se inferir que ambas as modalidades ventilatórias são seguras quanto à oxigenação arterial nessa espécie.

No entanto, ainda em relação à $\mathrm{PaO}_{2}$, em ambos os grupos, as médias em M0 foram menores que os valores registrados nos demais momentos. 
Resultados semelhantes foram relatados por Fontenelles et al. (2007), que verificaram aumento dessa característica após o choque hemorrágico em coelhos anestesiados com cetamina e xilazina, ventilados espontaneamente. Esses autores concluíram que o aumento da $\mathrm{PaO}_{2}$ é um sinal de compensação, devido à perfusão inadequada, levando a uma melhor utilização do oxigênio residual pelos tecidos.

Para $\mathrm{SaO}_{2}$, não houve diferença entre os grupos e ao longo do período experimental. Essa variável permaneceu estável e próxima aos valores considerados fisiológicos, 97 a $99 \%$ (Suckow e Douglas, 1996) para a espécie (Tab.2), indicando que ambas as técnicas ventilatórias foram capazes de manter a capacidade de oxigenação pulmonar adequada. Ademais, a maioria dos autores não tem observado alterações significativas na oxigenação dos pacientes quando a SIMV é comparada com outras modalidades ventilatórias (Matsumoto e Almeida, 2005). De acordo com Ferreira et al. (2005), a VAPSV mostrou ser segura quanto à oxigenação tecidual e trocas gasosas em pacientes humanos com insuficiência respiratória quando comparada com a ventilação controlada a volume ou a pressão.
Neste estudo, verificou-se que os valores da $\mathrm{PaCO}_{2}$ permaneceram dentro da faixa considerada normal para a espécie, 20 a 45 mmHg (Barzago et al., 1992). Tal estabilidade pode ser atribuída ao emprego da ventilação mecânica, que tem sido recomendada, durante os procedimentos anestésicos, para manter a concentração da $\mathrm{PaCO}_{2}$ próxima ao normal (Sá et al., 2010), pois a hipercapnia é um achado comum em coelhos submetidos à anestesia geral e hipovolemia, devido à depressão do centro respiratório (Sumpelmann et al., 2006; Fontenelles et al., 2007; Rezende Neto et al., 2010).

Em relação ao $\mathrm{pH}, \mathrm{HCO}_{3}^{-}$e $\mathrm{DB}$, não foram registradas diferenças significativas entre os grupos (Tab. 2), demonstrando que o emprego de diferentes modalidades ventilatórias não interferiram nesses parâmetros. Resultados semelhantes foram registrados por Sumplemann et al. (2006), que não observaram variações dessas variáveis em coelhos submetidos a hemorragia, anestesiados com cetamina e midazolam e ventilados mecanicamente.

Tabela 2. Valores médios e desvios padrão $(\mathrm{x} \pm \mathrm{s})$ de pressão parcial de oxigênio $\left(\mathrm{PaO}_{2}\right)$, pressão parcial de dióxido de carbono no sangue arterial $\left(\mathrm{PaCO}_{2}\right)$, saturação de oxihemoglobina no sangue arterial $\left(\mathrm{SaO}_{2}\right)$, déficit de base (DB), bicarbonato $\left(\mathrm{HCO}_{3}{ }^{-}\right)$e $\mathrm{pH}$ do sangue arterial obtidos em coelhos submetidos a hipovolemia aguda, anestesiados com propofol $(0,5 \mathrm{mg} / \mathrm{kg} / \mathrm{min})$ e ventilados com SIMV (GM) ou VAPSV (GV).

\begin{tabular}{ccccccccc}
\hline Parâmetros & Grupo & M0 & M1 & M2 & M3 & M4 & M5 & M6 \\
\hline $\mathrm{PaO} 2$ & GM & $127 \pm 22 \mathrm{a}$ & $139 \pm 25$ & $139 \pm 22$ & $152 \pm 28 \mathrm{~b}$ & $157 \pm 26 \mathrm{~b}$ & $160 \pm 26 \mathrm{~b}$ & $161 \pm 17 \mathrm{~b}$ \\
$(\mathrm{mmHg})$ & $\mathrm{GV}$ & $149 \pm 27 \mathrm{a}$ & $156 \pm 24 \mathrm{a}$ & $158 \pm 17 \mathrm{a}$ & $169 \pm 25$ & $183 \pm 27 \mathrm{~b}$ & $183 \pm 18 \mathrm{~b}$ & $183 \pm 22 \mathrm{~b}$ \\
$\mathrm{PaCO} 2$ & $\mathrm{GM}$ & $43 \pm 8$ & $44 \pm 8$ & $45 \pm 3$ & $46 \pm 8$ & $48 \pm 6$ & $48 \pm 6$ & $49 \pm 7$ \\
$(\mathrm{mmHg})$ & $\mathrm{GV}$ & $42 \pm 2$ & $43 \pm 5$ & $43 \pm 7$ & $44 \pm 3$ & $45 \pm 2$ & $46 \pm 8$ & $46 \pm 2$ \\
$\mathrm{SaO} 2$ & $\mathrm{GM}$ & $97 \pm 0,8$ & $97 \pm 0,9$ & $97 \pm 0,8$ & $97 \pm 0,7$ & $97 \pm 0,6$ & $97 \pm 0,5$ & $97 \pm 0,7$ \\
$(\%)$ & $\mathrm{GV}$ & $98 \pm 0,4$ & $98 \pm 0,5$ & $98 \pm 0,6$ & $98 \pm 0,3$ & $98 \pm 0,2$ & $98 \pm 0,3$ & $98 \pm 0,2$ \\
$\mathrm{pH}$ & $\mathrm{GM}$ & $7,26 \pm 0,03$ & $7,22 \pm 0,05$ & $7,21 \pm 0,03$ & $7,21 \pm 0,05$ & $7,21 \pm 0,06$ & $7,20 \pm 0,06$ & $7,20 \pm 0,03$ \\
$\mathrm{HCO}-$ & GV & $7,27 \pm 0,02$ & $7,23 \pm 0,05$ & $7,22 \pm 0,07$ & $7,22 \pm 0,03$ & $7,21 \pm 0,02$ & $7,20 \pm 0,02$ & $7,20 \pm 0,02$ \\
$(\mathrm{mEq} / \mathrm{L})$ & $\mathrm{GV}$ & $31 \pm 3$ & $30 \pm 3$ & $30 \pm 3$ & $30 \pm 5$ & $30 \pm 2$ & $30 \pm 3$ & $30 \pm 2$ \\
$\mathrm{DB}$ & GM & $3,9 \pm 3$ & $3,6 \pm 2$ & $3,5 \pm 3$ & $3,5 \pm 4$ & $3,5 \pm 6$ & $3,4 \pm 3$ & $3,3 \pm 2$ \\
$(\mathrm{mEq} / \mathrm{L})$ & GV & $3,6 \pm 2$ & $3,5 \pm 5$ & $3,3 \pm 5$ & $3,1 \pm 3$ & $3,0 \pm 0,2$ & $2,9 \pm 0,8$ & $2,9 \pm 1$ \\
\hline
\end{tabular}

Médias seguidas por letras minúsculas diferentes, nas linhas, diferem entre si com $\mathrm{P}<0,05$.

Vale ressaltar que, em condições fisiológicas, o pH sanguíneo do coelho é mais alcalino do que o do homem (Figueiredo et al., 2003), variando de 7,38 a 7,50 (Barzago et al., 1992). Contudo, nesta pesquisa, observou-se que, antes da hipovolemia, as médias dessa variável estavam abaixo do limite de normalidade, sugerindo acidose. Burow et al. (2004) e Barbosa (2007) relataram que a infusão contínua de propofol pode causar acidemia em pacientes humanos. Esses mesmos autores utilizaram como justificativa a inibição da atividade mitocondrial 
por redução da ação do citocromo C oxidase e/ou falha na oxidação dos ácidos graxos. Portanto, neste estudo, como não foi verificada em nenhum momento depressão respiratória ou hipóxia, a acidemia observada provavelmente ocorreu devido à administração contínua desse mesmo fármaco.

\section{CONCLUSÕES}

A SIMV e a VAPSV foram seguras quanto à oxigenação arterial, garantindo uma adequada troca gasosa. Contudo, a SIMV mostrou-se a mais indicada em coelhos hipovolêmicos por manter melhor a estabilidade hemodinâmica, com a vantagem de proporcionar menor trabalho respiratório.

\section{AGRADECIMENTOS}

Os autores agradecem à Fundação de Amparo à Pesquisa do Estado de São Paulo (FAPESP) pelo apoio financeiro concedido.

\section{REFERÊNCIAS}

AGUIAR, A.J.; LUNA, S.P.L.; OLIVA, V.N.L.S. et al. Continuous infusion of propofol in dogs premedicated with methotrimeprazine. Vet. Anaest. Analg., v.28, p.220-224, 2001.

ALEXANDER, D.J.; CLARK, G.C. A simple method of oral endotracheal intubation in rabbits (Oryctolagus cuniculus). Laboratory Anim. Sci., v.30, p.871-873, 1980.

BARBAS, C.S.V.; BUENO, M.A.S.; AMATO, M.B.P. et al. Interação cardiopulmonar durante a ventilação mecânica. Rev. Soc. Cardiol., v.8, p.406417, 1998 .

BARBOSA, F.T. Síndrome da infusão de propofol. Rev. Bras. Anestesiol., v.57, p.539-542, 2007.

BARZAGO, M.M.; BORTOLOTTI, A.; OMARINI, D. et al. Monitoring of blood gas parameters and acidbase balance of pregnant and non-pregnant rabbits (Oryctolagus cuniculus) in routine experimental conditions. Laboratory Anim., v.26, p.73-79, 1992.

BAURMET, J.H.; HECKER, K.E.; HEIN, M. et al. Haemodynamic effects of haemorrhage during xenon anaesthesia in pigs. Br. J. Anaesthesia, v.94 p.727732, 2005.
BONASSA, J.; AMATO, M.B.P. Ventilação volumétrica assistida com pressão suporte-VAPS. In: AULER, J.O.C.; AMARAL, R.V.G. (Eds). Assistência ventilatória mecânica. 1.ed. São Paulo: Atheneu, 1995. p.199-210.

BORGES, P.A.; NUNES, N.; CAMACHO, A.A. et al. Diferentes frações inspiradas de oxigênio em coelhos hipovolêmicos anestesiados com propofol e submetidos à ventilação mecânica. Cienc. Rural, v.41, p.1960-1966, 2011.

BUROW, B.K.; JOHNSON, M.E.; PACKER, D.L. et al. Metabolic acidosis associated with propofol in the absence of other causative factors. Anesthesiology, v.101, p.239-241, 2004.

CORTOPASSI, S.R.G. Complicações da anestesia. In: FANTONI, D.T; CORTOPASSI, S.R.G. (Eds). Anestesia em cães e gatos. São Paulo: Roca, 2002. cap. 33, p.347-361.

DAVID, C.M. Ventilação mecânica: da fisiologia à prática clínica. Rio de Janeiro: Revinter, 2001. 528p.

DENEUCHE, A.; DESBOIS, C. Propofol 1Pharmacological features. Le Point Vét., v.30, p.29-34, 1999.

EMMERICH, J.C. Início e manutenção do suporte ventilatório. In: EMMERICH, J.C. (Ed). Suporte ventilatório. Aplicação prática. 3 ed. Rio de Janeiro: Revinter, 2008. p.21-41.

EMMERICH, J.C. Modalidades ventilatórias e duplo controle e em alça fechada. In: DAVID, C.M.N. (Ed). Medicina Intensiva. Rio de Janeiro: Revinter, 2001. p.429-439.

FERREIRA， C.J.; VALIATTI, J.; SCHETTING, G.P.P. et al. Comparação do Modo VAPS com os Modos Volume Controlado e Pressão Controlada em Pacientes com Insuficiência Respiratória Aguda. Rev. Bras. Terapia Intensiva, v.17, p.89-93, 2005.

FIGUEIREDO, N.V.; SHANAIDER, A.; BARRUCAND, L. et al. Hipotensão controlada induzida por halotano em coelhos. Rev. Col. Bras. Cirurgia, v.30, p.344-355, 2003.

FINK, M.P. Shock: An overview. In: RIPPE, J.M.; INVIN, R.S.; FINK, M.P. et al. (Eds). Intensive Care Medicine. 3th ed. Little, Brown and Company, 1996. p.1857-1877.

FLECKNELL, P.A. Laboratory animals. In: TRANQUILLI, W.J.; THURMON, J.C. (Eds). Lumb \& Jones' veterinary anesthesia and analgesia. 4th ed. Oxford: Blackwell Publishing, 2007. p.765-784.

FONTENELES, M.J.; CARVALHO, R.M.; OLIVEIRA, L.M.R. et al. Profile of Hemodynamic and Gasometric Parameters in Rabbits Submitted to Controleed Hemorrhagic Shock. Rev. Paraense de Medicina, v.21, p.15-21, 2007. 
HASKINS, S.C. The effect of moderate hipovolemia on cardiopulmonary function in dogs. J. Vet. Emergency and Critical Care, v.15, p.100-109, 2005.

HIRANO, E.S.; MANTONANI, M.; MORANDIN, R.C. et al. Modelo experimental de choque hemorrágico. Acta Cirúrgica Bras., v.18, p.465-469, 2003.

LEVISTZKY, M.G. Difusão de gases. In: LEVISTZKY, M.G. (Ed). Fisiologia pulmonar. São Paulo: Manole. 2004, p.130-141.

LOPES, P.C.F.; NUNES, N.; SOUSA, M.G. et al. Efeitos de diferentes $\mathrm{FiO}_{2}$ sobre variáveis ecocardiográficas em cães submetidos à infusão contínua de propofol. Arq. Bras. Med. Vet. Zootec., v.61, p.345-352, 2009.

MANCZUR, T.; GREENOUGH, A. Comparison of the pressure time product during synchronous intermittent mandatory ventilation and contínuos positive airway pressure. Arch. Dise. Childhood, v.83, p.265-267, 2000.

MARTINS, C.C.; BOTTI, J.V.; CASTRO, L.T. et al. Comparação entre três métodos de desmame gradual da ventilação mecânica. Rev. Cientifica Famina, v.1, p.13-30, 2005.

MATSUMOTO, T.; ALMEIDA, N.M. Ventilação mandatória intermitente. In: CARVALHO, B.W. et al. (Eds). Ventilação pulmonar mecânica em pediatria e neonatologia. 2.ed. São Paulo: Atheneu, 2005. v.16, p.165-171.

MORO, J.V. Efeitos cardiovasculares e respiratórios da infusão contínua de naloxona tramadol, em coelhos anestesiados com isofluorano e submetidos à hipovolemia aguda.. 57f. Tese (Mestrado em Cirurgia veterinária) - Faculdade de Ciências Agrárias e Veterinárias, Universidade Estadual Paulista, Jaboticabal, 2009.

NEUMANN, P.; WRIGGE, H.; ZINSERLING, J. et al. Spontaneous breathing affects the spatial ventilation and perfusion distribution during mechanical ventilatory support. Crit Care Med., v.33, p.1090-1095, 2005.
NOLAN, J.P.; PARR, J.A. Aspects of resuscitation in trauma. Brit. J. Anaest., v.79, p.226-240, 1997.

PEPE, P.A.; LURIE, K.G.; WIGGINTON, J.G. Detrimental hemodynamic effects of assisted ventilation in hemorrhagic states. Crit. Care Med., v.32, p.414-420, 2004.

REVES, J.G. Nonbarbiturate intravenous anesthetic. In: MILLER, R. (Ed). Anesthesia 5th ed. Philadelphia: Churchill Linvstone, 2000. p.228-272.

REZENDE-NETO, J.B.; RIZOLI, S.B.; ANDRADE, M.V. et al. Rabbit model of uncontrolled hemorrhagic shock and hypotensive resuscitation. Braz. J. Med. Biol. Res., v.43, p.1153-1159, 2010.

ROMALDINI, H. Repercussões Cardiovasculares da Ventilação Mecânica. In: AULER JUNIOR, J.O.C.; AMARAL, R.V.G. (Eds). Assistência Ventilatória Mecânica. São Paulo: Atheneu. 2006. p.115-121.

ROYSE, C.F. Persistent depression of contractility and vasodilation with propofol but not with sevoflurane or desflurane in rabbits. Anesthesiology, v.108, p.87-93, 2008.

SÁ, P.A.; TEIXEIRA NETO, F.J.; CAMPAGNOL, D. et al. Efeitos do modo ventilatório sobre variáveis hemogasométricas em equinos submetidos à mudança de decúbito durante a anestesia geral inalatória com halotano. Arq. Bras. Med. Vet. Zootec., v.62, p.549$554,2010$.

SUCKOW, M.A.; DOUGLAS, F.A. The Laboratory Rabbit. Important Biological Features. In: SUCKOW, M.A. (Ed). Veterinary care. 2nd ed. Flórida: Boca Raton. 1996. p.1-8.

SUMPELMANN, R.; SCHUERHOLZ, T.; MARX, G. et al. Haemodynamic, acid-base and blood volume changes during prolonged low pressure pneumoperitoneum in rabbits. Br. J. Anaesthesia, v.96, p.563-568, 2006. 